\title{
Phase Noise Analysis for a mm-Wave VCO configuration
}

\author{
Deepa George ${ }^{\dagger}$, Graduate student member, IEEE, deepag@ieee.org, and \\ Saurabh Sinha ${ }^{\dagger \dagger}$, SMIEEE, ssinha@ieee.org \\ ${ }^{\dagger}, \dagger$ Department of Electrical, Electronic and Computer Engineering, University of Pretoria, South Africa \\ University of Pretoria, Lynnwood Road, Hillcrest, Pretoria, South Africa, 0002
}

\begin{abstract}
The letter highlights the importance of modelling the phase noise of an oscillator using the impulse sensitivity function. A Colpitts oscillator in the common collector configuration is analyzed to obtain an expression for its phase noise. The oscillator design is thus optimized for phase noise with respect to process and design parameters. The fabricated voltage controlled oscillator at an oscillating frequency of $52.8 \mathrm{GHz}$ has a measured phase noise of $-\mathbf{9 8 . 9} \mathrm{dBc} / \mathrm{Hz}$ at $1 \mathrm{MHz}$ offset.
\end{abstract}

Key words —mm-wave, MMIC, SiGe heterojunction bipolar transistor, VCO, phase noise

\section{INTRODUCTION}

The IEEE 802.15.3 Task Group 3c (TG3c) has developed a millimetre-wave (mm-wave) based physical layer for the wireless personal area network standard, which operates in the 57-64 GHz unlicensed band and can provide high data rates over 2 Gbps. Voltage-controlled oscillators (VCOs) with improved phase noise performance are mandatory for any high-performance wireless receiver system, as they determine the system's sensitivity. A proper estimate of phase noise could be obtained by modelling the phase noise in terms of its design and process parameters [1]. In this letter, phase noise analysis is performed on a common collector configuration of the Colpitts oscillator by using the impulse sensitivity function (ISF) [2]. The phase noise is analytically derived by studying the contribution of major noise sources to the phase noise and quantifying this, using the ISF of the corresponding noise source. The analysis is verified by a VCO design at $60 \mathrm{GHz}$ using the IBM BiCMOS 8HP process, which is a SiGe heterojunction bipolar transistor (HBT) technology with a $f_{T}$ of $200 \mathrm{GHz}$.

\section{VOLTAGE-CONTROLLED OSCILLATORS}

The high-performance mm-wave VCO implementations with the lowest phase noise have been reported for a differential common-collector Colpitts oscillator in SiGe technology [3][4][5]. The 
common collector configuration is preferred at higher frequencies because of its better stability, as the tank is buffered from the load by the transistors $Q_{l}$ (or $Q_{2}$ ), as shown in Figure 1(a). SiGe has been the choice of technology over CMOS for low-phase noise oscillators [6] owing to its lower $1 / f$ noise, which is up-converted into phase noise of the oscillator in the $1 / f^{3}$ region. A differential configuration is preferred in the mm-wave range to minimize the generation and coupling of high-frequency signals. It also has the advantage of less criticality to on- and off-chip decoupling of supply and bias voltages due to the virtual ground node [7].

The basic core of a differential common collector Colpitts oscillator comprises two half circuits consisting of $Q_{1}, L_{b}, C_{1}$ and $C_{2}$ as shown in Figure 1(a). $R_{T}$ models the total tank resistance, mainly contributed by the passives in the tank, and $I_{B I A S}$ is the bias current for a half circuit.

The resonator tank voltage $A_{\text {tank }}$ in Figure 1(b) is given by the fundamental tank current $I_{l}$, the total tank resistance $R_{T}$ and the equivalent impedance transformation ratio $n$

$$
A_{\text {tank }}=I_{1} R_{T}(1-n)
$$

where $n$ is the ratio of the capacitors given by

$$
n=\frac{C_{2}}{C_{1}+C_{2}}
$$

Then $A_{e}$, the base-emitter junction ac voltage is given by

$$
A_{e} \approx n A_{\text {tank }}
$$

If $V_{B E}$ is the dc voltage across the junction, then the collector current is given by

$$
I_{c}(\phi)=I_{s} e^{\frac{V_{B E}+A_{e} \cos \phi}{V_{T}}}
$$

where $I_{s}$ is the BJT saturation current and

$$
V_{T}=\frac{k_{B} T}{q}
$$


where $k_{B}=8.617385 \times 10^{-5} \mathrm{eV} / \mathrm{K}$ is the Boltzmann's constant and $T$ is the absolute temperature.

The average dc value of the transistor current over a cycle should be equal to the bias current $I_{B I A S}$

$$
I_{B I A S}=\frac{1}{2 \pi} \int_{-\pi}^{+\pi} I_{c}(\phi) d \phi==I_{s} e^{\frac{V_{B E}}{V_{T}}} B_{0}\left(a_{e}\right)
$$

where $B_{0}\left(a_{e}\right)$ is a modified Bessel function of the first kind given by

$$
\begin{gathered}
B_{m}(x)=\frac{1}{\pi} \int_{0}^{\pi} e^{x \cos t} \cos (m t) d t \\
a_{e}=\frac{A_{e}}{V_{T}}
\end{gathered}
$$

The first harmonic $I_{l}$ of the transistor current is given by Fourier's theory as

$$
I_{1}=\frac{1}{\pi} \int_{-\pi}^{+\pi} I_{c}(\phi) \cos (\phi) d \phi=2 I_{s} e^{V_{B E} / V_{T}} \cdot B_{1}\left(a_{e}\right)=2 I_{B I A S} \frac{B_{1}\left(a_{e}\right)}{B_{0}\left(a_{e}\right)}
$$

If $a_{e}$ is large, then the fundamental current $I_{l}$ can be approximated as

$$
I_{1} \approx 2 I_{B I A S}
$$

\section{PHASE NOISE ANALYSIS}

In this section, phase noise analysis is performed on a common-collector configuration of the Colpitts oscillator using ISF theory by identifying dominant noise sources in the circuit. The phase noise expression was derived by combining the contribution from various identified noise sources. The ISF of an individual noise source is calculated by injecting current impulse in parallel to the noise source and its effect on the tank capacitance is evaluated by using small-signal model circuit analysis.

As found by [2], the tank resistance $R_{T}$ causes a phase noise at offset frequency $\Delta \omega$ given by

$$
L(\Delta \omega)=10 \log \left(\frac{\Gamma_{R_{T, m m s}}^{2}}{q_{\max }^{2}} \cdot \frac{\overline{i_{R_{T}}^{2}}}{2 \Delta \omega^{2}}\right)=10 \log \left(\frac{\Gamma_{R_{T, m s}}^{2}}{A_{\text {tank }}^{2} C^{2}} \cdot \frac{\overline{i_{R_{T}}^{2}}}{2 \Delta \omega^{2}}\right)
$$


where $q_{\max }$ is the maximum charge across the capacitance in an oscillation period, which could be substituted with the product of tank voltage $A_{\text {tank }}$ and tank capacitance $C ; \bar{i}_{R_{T}}^{2} / \Delta f$ is the power spectral density of white noise generated by the tank resistance $R_{T}$ given by

$$
\frac{\overline{i_{R_{T}}^{2}}}{\Delta f}=\frac{4 k_{B} T}{R_{T}}
$$

and $\Gamma_{R_{T}}$ is the ISF of the noise source due to tank resistance $R_{T}$. The $\Gamma_{R_{T}}$ for an LC tank differential oscillator is given by

$$
\Gamma_{R_{T}}(\phi)=\frac{\sin (\phi)}{N}
$$

where $N=1$ for a single-ended oscillator and $N=2$ for a differential oscillator.

The base current shot noise contribution is neglected, as it is very small [8]. The contribution of collector current shot noise and base resistance thermal noise is evaluated by using their ISF. In this work, the approach followed to find the ISF of any noise source is to relate it to the ISF of tank resistance, by evaluating the voltage change at the tank capacitance caused by the respective current impulses, as shown in the subsections below.

\subsection{Phase Noise by Collector Current Shot Noise}

When a cyclo-stationary noise source such as the collector current shot noise in Figure 2(a) is considered, (11) has to be modified to include the effective ISF $\Gamma_{i_{c}, \text { eff }}$ given by

$$
\Gamma_{i_{c}, e f f}(\phi)=\Gamma_{i_{c}}(\phi) \cdot \alpha_{i_{c}}(\phi)
$$

where the dependence of noise power on $\phi$ is taken into account by $\alpha_{i_{c}}(\phi)$.

$\Gamma_{i_{c}}(\phi)$ is found by injecting a current impulse of area $\Delta Q$ into the same oscillator node where the noise current flows as in Figure 2(b). 
In Figure 2(b), the voltage change $\Delta V_{R_{T}}$ across the tank capacitor caused by a current impulse of area $\Delta Q$ injected in parallel to the tank resistance at node $\mathrm{A}$ is given by

$$
\Delta V_{R_{T}}=\frac{\Delta Q}{C}
$$

where

$$
C=\frac{C_{1} C_{2}}{C_{1}+C_{2}}
$$

The voltage $\Delta V_{A, B}$ measured at node $\mathrm{A}$, generated by a current impulse flowing into node $\mathrm{B}$, is given by

$$
\Delta V_{A, B}=\frac{\Delta Q}{C_{2}}=\frac{\Delta V_{R_{T}} C}{C_{2}}=\Delta V_{R_{T}} \frac{C_{1}}{C_{1}+C_{2}}=(1-n) \Delta V_{R_{T}}
$$

Hence the ISF $\Gamma_{i_{c}}$ is related to $\Gamma_{R_{T}}$ according to (17) and substituting (13) gives

$$
\Gamma_{i_{c}}=(1-n) \Gamma_{R_{T}}=(1-n) \frac{\sin (\phi)}{N}
$$

The periodicity information $\alpha_{i_{c}}(\phi)$ for calculating the effective ISF in (14) is obtained from the expression of collector current shot noise [7] and is given by

$$
\alpha_{i_{c}}(\phi) \equiv \sqrt{e^{a_{e} \cos (\phi)}}
$$

Thus $\Gamma_{i_{c}, \text { eff }}(\phi)$ in (14) could be re-written using (18) and (19) as

$$
\Gamma_{i_{c}, e f f}(\phi)=\Gamma_{i_{c}}(\phi) \cdot \alpha_{i_{c}}(\phi)=(1-n) \frac{\sin (\phi)}{N} \sqrt{e^{a_{e} \cos (\phi)}}
$$

This gives the expression for $L_{i_{c}}(\Delta \omega)$, the phase noise contribution of the collector current shot noise as

$$
L_{i_{c}}(\Delta \omega)=10 \log \left(\frac{k_{B} T(1-n)}{2 N R_{T} n C^{2} A_{\text {tank }}^{2} \Delta \omega^{2}}\right)
$$




\subsection{Phase Noise by Base Resistance Thermal Noise}

The noise contribution by the base resistance thermal noise in Figure 3 is calculated by relating the ISF of the noise source $\Gamma_{r_{b}}(\phi)$ to the ISF of the tank resistance $\Gamma_{R_{T}}(\phi)$

$$
\Gamma_{r_{b}}(\phi)=\frac{\Delta V_{r_{b}}(\phi)}{\Delta V_{R_{T}}(\phi)} \Gamma_{R_{T}}(\phi)
$$

where $\Delta V_{r_{b}}(\phi)$ is the voltage change across the tank capacitor caused by injecting a current impulse $\Delta Q$ parallel to $r_{b}$ and as defined earlier, $\Delta V_{R_{T}}(\phi)$ is the voltage change across the capacitor caused by injecting a current impulse parallel to $R_{T}$ and given by

$$
\Delta V_{R_{T}}=\frac{\Delta Q}{C}
$$

As $\Delta V_{r_{b}}(\phi)$ and $\Delta V_{R_{T}}(\phi)$ are both evaluated across the same tank capacitance, the ISF could be rewritten as

$$
\Gamma_{r_{b}}(\phi)=\frac{\Delta Q_{r_{b}}(\phi)}{\Delta Q(\phi)} \Gamma_{R_{T}}(\phi)
$$

The analysis is done by assuming a negligible parasitic capacitance at the base, $C_{B}$ to the ground as shown in Figure 3 and injecting a charge impulse in parallel to $r_{b}$ at time $t_{0} . C_{B}$ is in series with a relatively large tank capacitance $C$; hence the charge in the current impulse is collected by $C_{B}$ and is discharged through the network $C_{B^{-}} r_{b}-C$ with a time constant $\tau=r_{b} C_{B}$ as $C_{B}<<C$. The voltage at the base of the transistor is given by

$$
V_{C_{B}}(t)=\frac{\Delta Q}{C_{B}} e^{-\frac{\left(t-t_{0}\right)}{\tau}}
$$

This leads to an impulsive current given by the transistor transconductance and the base voltage according to 


$$
\Delta I(t)=g_{m}\left(\omega_{0} t\right) V_{C_{B}}(t)=g_{m}\left(\omega_{0} t\right) \frac{\Delta Q}{C_{B}} e^{-\frac{\left(t-t_{0}\right)}{\tau}}
$$

It could be noted from Figure 3 that the ISF of the impulsive current $\Delta I(t)$ will be similar to that of the collector current shot noise $\Gamma_{i_{c}}(\phi)$ in Figure 2(a). The charge in the impulsive current $\Delta I(t)$ could be found as follows:

$$
\Delta Q_{r_{b}}^{\prime}(\phi)=\int_{t_{0}}^{\infty} \Delta I(t) d t=g_{m}(\phi) r_{b} \Delta Q(\phi)
$$

The ISF associated with the base resistance thermal noise $\Gamma_{r_{b}}(\phi)$ is given by

$$
\begin{gathered}
\Gamma_{r_{b}}(\phi)=\frac{\Delta Q_{r_{b}}(\phi)}{\Delta Q(\phi)} \Gamma_{R_{T}}(\phi)=\left[\frac{\Delta Q_{r_{b}}(\phi)}{\Delta Q_{r_{b}}^{\prime}(\phi)} \Gamma_{R_{T}}(\phi)\right] \frac{\Delta Q_{r_{b}}^{\prime}(\phi)}{\Delta Q(\phi)} \\
\Gamma_{r_{b}}(\phi)=\Gamma_{i_{c}}(\phi) \frac{\Delta Q_{r_{b}}^{\prime}(\phi)}{\Delta Q(\phi)}=\Gamma_{i_{c}}(\phi) g_{m}(\phi) r_{b}
\end{gathered}
$$

Substituting (18) for $\Gamma_{i_{c}}(\phi)$ would give the following expression for $\Gamma_{r_{b}}(\phi)$

$$
\Gamma_{r_{b}}(\phi)=(1-n) \frac{\sin (\phi)}{N} g_{m}(\phi) r_{b}
$$

This expression for $\Gamma_{r_{b}}(\phi)$ is the same as that for a common base configuration, hence the phase noise due to the base resistance thermal noise is given by

$$
L_{r_{b}}(\Delta \omega)=10 \log \left(\frac{k_{B} T I_{B I A S} r_{b}(1-n)}{2 N V_{T} R_{T} n C^{2} A_{\text {tank }}^{2} \Delta \omega^{2}} \cdot \frac{B_{1}\left(2 a_{e}\right)}{B_{0}\left(a_{e}\right) B_{1}\left(a_{e}\right)}\right)
$$

Thus the total phase noise for the common collector configuration is obtained by adding the individual contribution and is given by

$$
L_{t o t}(\Delta \omega)=10 \log \left\{\frac{k_{B} T(1+n)}{2 N R_{T} n C^{2} A_{\text {tank }}^{2} \Delta \omega^{2}} \cdot\left[1+\left(\frac{I_{B I A S} r_{b}(1-n)}{V_{T}(1+n)} \cdot \frac{B_{1}\left(2 a_{e}\right)}{B_{0}\left(a_{e}\right) B_{1}\left(a_{e}\right)}\right)\right]\right\}
$$


The above analysis shows that the expression for the phase noise in a common collector configuration is exactly the same as that for a common-base configuration [8]. Thus it could be concluded that the process parameter contribution to phase noise is independent of oscillator configuration or the way in which the LC tank is connected around the transistor.

The optimum value of the design parameter $n$ for a mm-wave oscillator could be determined by plotting the phase noise for different values of $n$ after substituting the following typical values in (32) $T=310 \mathrm{~K} ; f_{\text {osc }}=60 \mathrm{GHz} ; I_{B I A S}=18 \mathrm{~mA} ; C=142 \mathrm{fF} ; R_{T}=100 \Omega$ for a tank $Q=6$ (typical in mm-wave region); $r_{b}=12 \Omega$.

The optimal value of $n$ is approximately 0.3 , as is evident from the plot in Figure 4, and this is taken into account in the VCO design below.

\section{CIRCUIT DESIGN}

The process chosen for the design and fabrication of the mm-wave integrated circuit (MMIC) is the IBM BiCMOS $8 \mathrm{HP}$ process. The process offers high performance varactors and transmission lines necessary for VCO implementation. To reduce the design cycle time, only the standard components available in the process were used for the design. The simulations were performed using the devices characterized as p-cells and made available to the designer by the foundry. A cascode topology with a basic oscillator and a cascode buffer, as shown in Figure 5, was chosen for better isolation and stability.

In Figure 5, the tank inductors $L_{b}$ at the base of the transistors $Q_{1}$ (or $Q_{2}$ ) are presented with a negative real part and a capacitive reactance. This reactance is influenced by the varactor, $C_{V A R}$ and helps in tuning the oscillation frequency. $Q_{3}$ and $Q_{4}$ form the cascode transistors to buffer the tank from the load. As $Q_{3}$ (or $Q_{4}$ ), is separated from the tank by $Q_{1}$ (or $Q_{2}$ ), its impact on the phase noise is minimal [9]. The inductors $L_{c}$ at the collector of $Q_{3}$ and $Q_{4}$ form the output network designed to resonate at $60 \mathrm{GHz}$. Inductors $L_{e}$ are placed in the emitter branch of the circuit to implement the LC filtering technique to reduce the phase noise contribution of the current source [10]. $Q_{5-9}$ and $R_{1-6}$ constitute the bias network of the circuit. The transistor size was chosen to be $W=0.12 \mu \mathrm{m}$ and $L=18$ 
$\mu \mathrm{m}$ (the width was fixed for the process and the length was the largest available in the process), as higher-sized transistors have lower extrinsic and intrinsic resistances, which in turn reduces the thermal noise contribution to phase noise (32)

The circuit has three inductors in each of the half circuit, the tank inductance $L_{b}$, the inductors in the output network $L_{c}$ and the inductors used for phase noise minimization, $L_{e} . L_{b}$ is realized using microstrip lines available in the process. $L_{c}$ and $L_{e}$ are realized using line inductors in the process. As transistor parasitics affect the oscillating frequency, the design required adjustment of the values of capacitors $C_{1}$ and $C_{2}$ to maintain a center frequency of $60 \mathrm{GHz}$ for varying $n$, as shown in Figure 6 . Phase noise simulations were then performed to obtain the optimal value of the design parameter $n$ that gives the lowest phase noise.

The phase noise simulations in Figure 6 give the minimum value of phase noise for $n$ as approximately 0.3 , which was also what was obtained using the theoretical analysis (Figure 4).

As phase noise improves with quality factor, a high $Q$ LC tank is the primary requirement for a lownoise design. In the mm-wave frequency range, varactor $Q$ is seen as the primary factor determining the quality factor of the tank. HBT varactors have better quality factor and tuning range than their MOS counterparts in the mm-wave range, leading to using HBT varactors in the implementation of VCO. Varactor $Q$ also improves with reduced width and increased number of base stripes, as they minimize the base resistance. High quality metal-insulator-metal capacitors are used as the fixed capacitor $C_{1}$ in Figure 5.

\section{MEASURED PERFORMANCE}

The chip was probed on wafer-level to measure the performance, as packaging degrades circuit performance at mm-wave frequencies because of the inductive effects of wirebonds. A typical impedance of $12 \Omega+\mathrm{j} 360 \Omega$ is presented at $60 \mathrm{GHz}$ if a $1 \mathrm{~mm}$ long wirebond is modelled as a $1 \mathrm{nH}$ inductor with a $Q$ of 30 [11]. A photograph of the chip is shown in Figure 7. 
The chip was attached to ground-signal-ground probes on either side. One side was terminated with a $50 \Omega$ termination and the other was connected to the channel of a spectrum analyzer for measurements. The spectrum analyzer used was Anritsu MS2668C (9 kHz - $40 \mathrm{GHz})$, extended to operate for 50$75 \mathrm{GHz}$ by connecting an external V-band mixer (MA2744A).

The VCO operated from a voltage of $4 \mathrm{~V}$ and consumed $140 \mathrm{~mW}$. The spectrum analyzer output at a center frequency of $52.8 \mathrm{GHz}$ and the corresponding phase noise value of $-98.9 \mathrm{dBc} / \mathrm{Hz}$ at $1 \mathrm{MHz}$ offset was observed as shown in Figure 8.

The tuning voltage was varied from $2.5 \mathrm{~V}$ to $6.5 \mathrm{~V}$ and a tuning range of $50 \mathrm{GHz}-57 \mathrm{GHz}$ was observed. The simulated and measured tuning characteristics are shown in Figure 9.

The difference in tuning characteristics in Figure 9 is due to interconnect parasitics and process variations, which brought about the $10 \%$ reduction in the tuning range available from the tank.

\section{Conclusion}

The phase noise analysis using ISF theory was performed on a Colpitts oscillator in the commoncollector configuration. The noise sources in the circuit, namely the tank losses, collector current shot noise and base resistance thermal noise were analyzed. The resultant closed-form expression for phase noise was found to be the same as that for a common-base configuration. The optimum value of the capacitance ratio $n$ was found to be 0.3 and the theoretical analysis was verified with simulations using SpectreRF. A $60 \mathrm{GHz}$ VCO was designed and fabricated using the $0.13 \mu \mathrm{m}$ IBM $8 \mathrm{HP}$ process and the measurement results were presented. 


\section{REFERENCES}

[1] A. Bevilacqua and P. Andreani, Phase noise analysis of the tuned-input-tuned-output (TITO) oscillator, IEEE Trans Circuits Syst II: Express Briefs 59 (2012), 20-24.

[2] A. Hajimiri and T.H. Lee, A general theory of phase noise in electrical oscillators, IEEE J Solid-State Circuits 33 (1998), 179-194.

[3] H. Li, H.-M. Rein, R. Kreienkamp and W. Klein, $47 \mathrm{GHz}$ VCO with low phase noise fabricated in a SiGe bipolar production technology, IEEE Microw Wireless Compon Lett 12 (2002), 79-81.

[4] N. Pohl, H.-M. Rein, T. Musch, K. Aufinger and J. Hausner, SiGe bipolar VCO with ultrawide tuning range at $80 \mathrm{GHz}$ center frequency, IEEE J Solid-State Circuits 44 (2009), 26552662.

[5] A. Barghouthi and F. Ellinger, Design of a 54 to $63 \mathrm{GHz}$ differential common collector SiGe colpitts, $18^{\text {th }}$ International Conference on Microwave, Radar and Wireless Communications, Dresden, Germany, 2010, pp 1-4.

[6] K.W. Hamed, A.P. Freundorfer and Y.M.M. Antar, An integrated 24-GHz differential VCO using SiGe HBT technology, Microw Opt Technol Lett 50 (2008), 2322-2325.

[7] H. Li and H.-M. Rein, Millimeter-wave VCOs with wide tuning range and low phase noise, fully integrated in a SiGe bipolar production technology, IEEE J Solid-State Circuits 38 (2003), 184-191.

[8] A. Fard and P. Andreani, An analysis of $1 / \mathrm{f}^{2}$ phase noise in bipolar colpitts oscillators (with a digression on bipolar differential-pair LC oscillators), IEEE J Solid-State Circuits 42 (2007), 374-384.

[9] S.P. Voinigescu, D. Marchesan and M.A. Copeland, A family of monolithic inductor-varactor SiGe-HBT VCOs for $20 \mathrm{GHz}$ to $30 \mathrm{GHz}$ LMDS and fiber-optic receiver applications, IEEE 
Radio Frequency Integrated Circuits (RFIC) Symposium, Boston, USA, 2000, pp 173-177.

[10] E. Hegazi, H.Sjoland and A. Abidi, A filtering technique to lower LC oscillator phase noise, IEEE J Solid-State Circuits 36 (2001), 1921-1930.

[11] A. Hajimiri, Holistic design in mm-wave silicon ICs, IEICE Trans on Electronics E91.C (2008), 817-828.

Figure legends

Figure 1 (a) Schematic of a differential common-collector Colpitts oscillator (b) Equivalent large signal model of its half circuit for calculating the tank amplitude

Figure 2 (a) Collector current shot noise in an oscillator (b) Circuit to calculate the ISF of collector current shot noise

Figure 3 Circuit to calculate the ISF of base resistance thermal noise

Figure 4 Phase noise for different values of $n$

Figure 5 Circuit schematic of the $60-\mathrm{GHz}$ VCO

Figure 6 Capacitances for an oscillating frequency of $60 \mathrm{GHz}$ and their simulated phase noise

Figure 7 Photograph of the 60-GHz VCO MMIC

Figure 8 Spectrum analyzer output at a center frequency of $52.8 \mathrm{GHz}$.

Figure 9 Measured and simulated tuning characteristics of the $60-\mathrm{GHz}$ VCO 
Figure 2

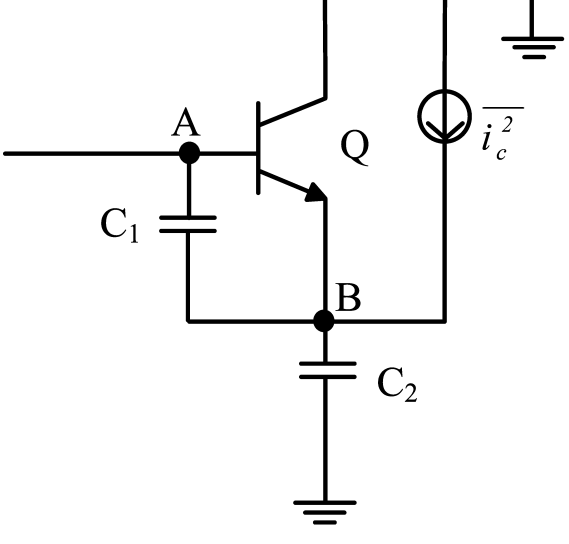

(a)

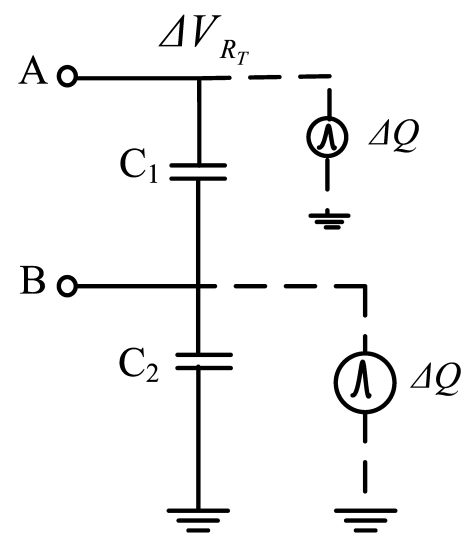

(b) 
Figure 4

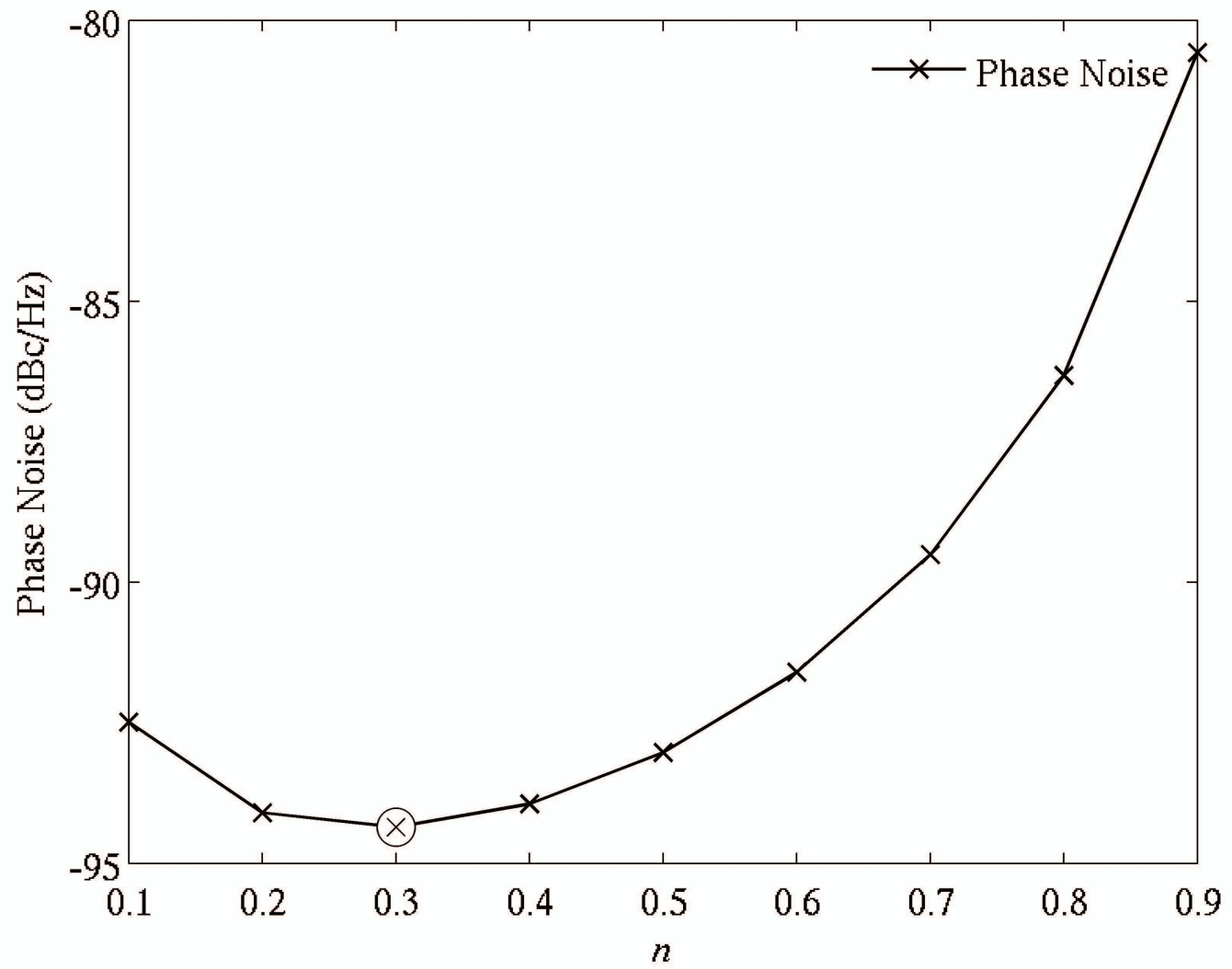




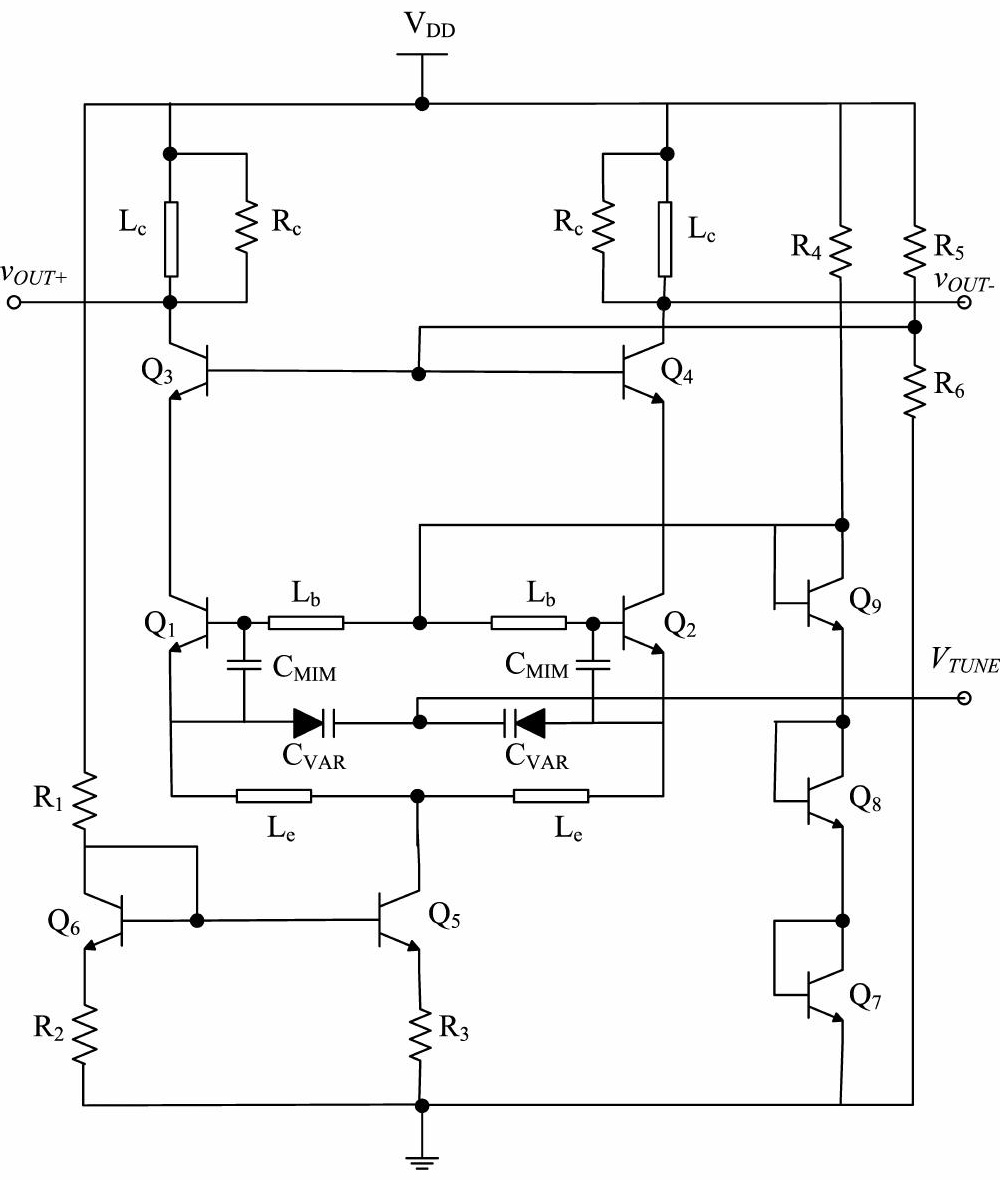




\section{Figure 6}

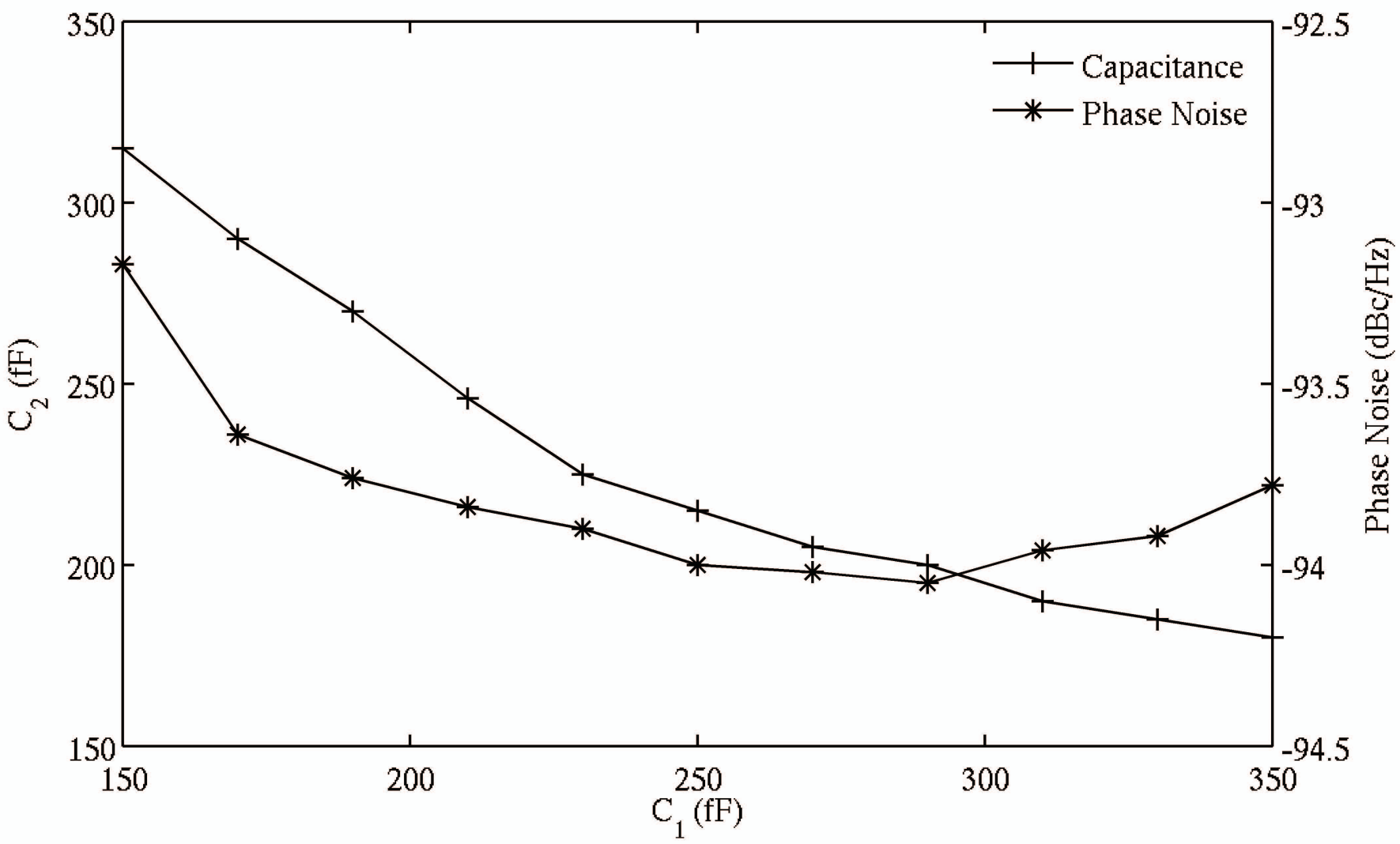


Figure 7

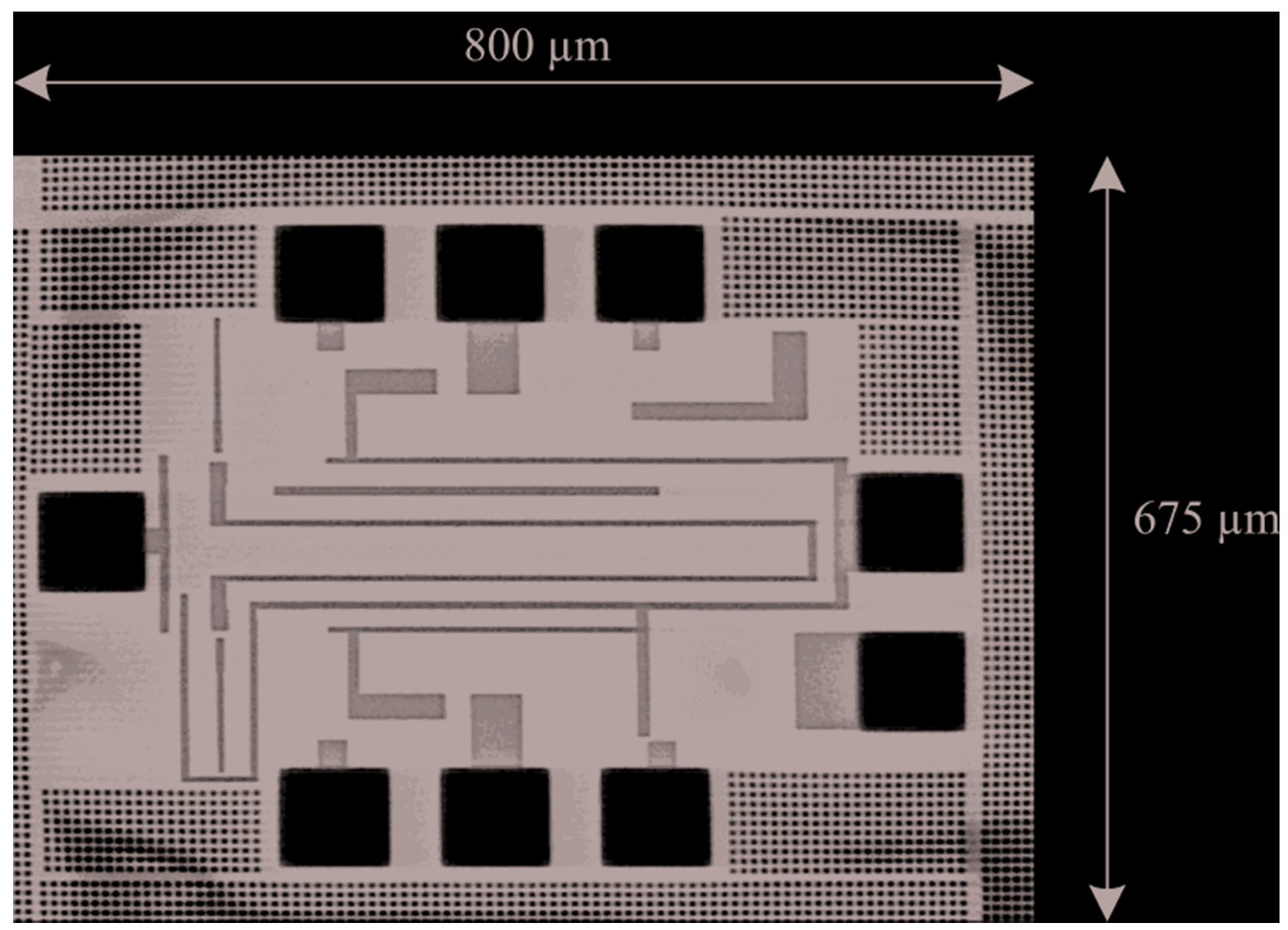


DLT: $1.00 \mathrm{MHz}$ $-49.68 \mathrm{~dB}$

RLV: -18.30dBm
Figure 8

09/04/2012

RB 100khz

VB 10khz
18:17:57

AT Obd

ST $50 \mathrm{~ms}$

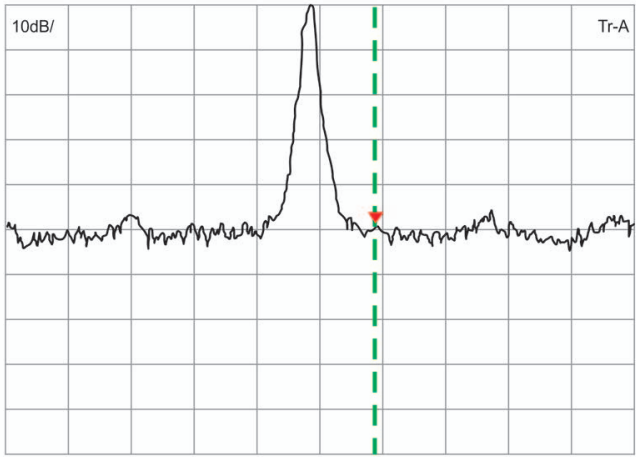


Figure 9

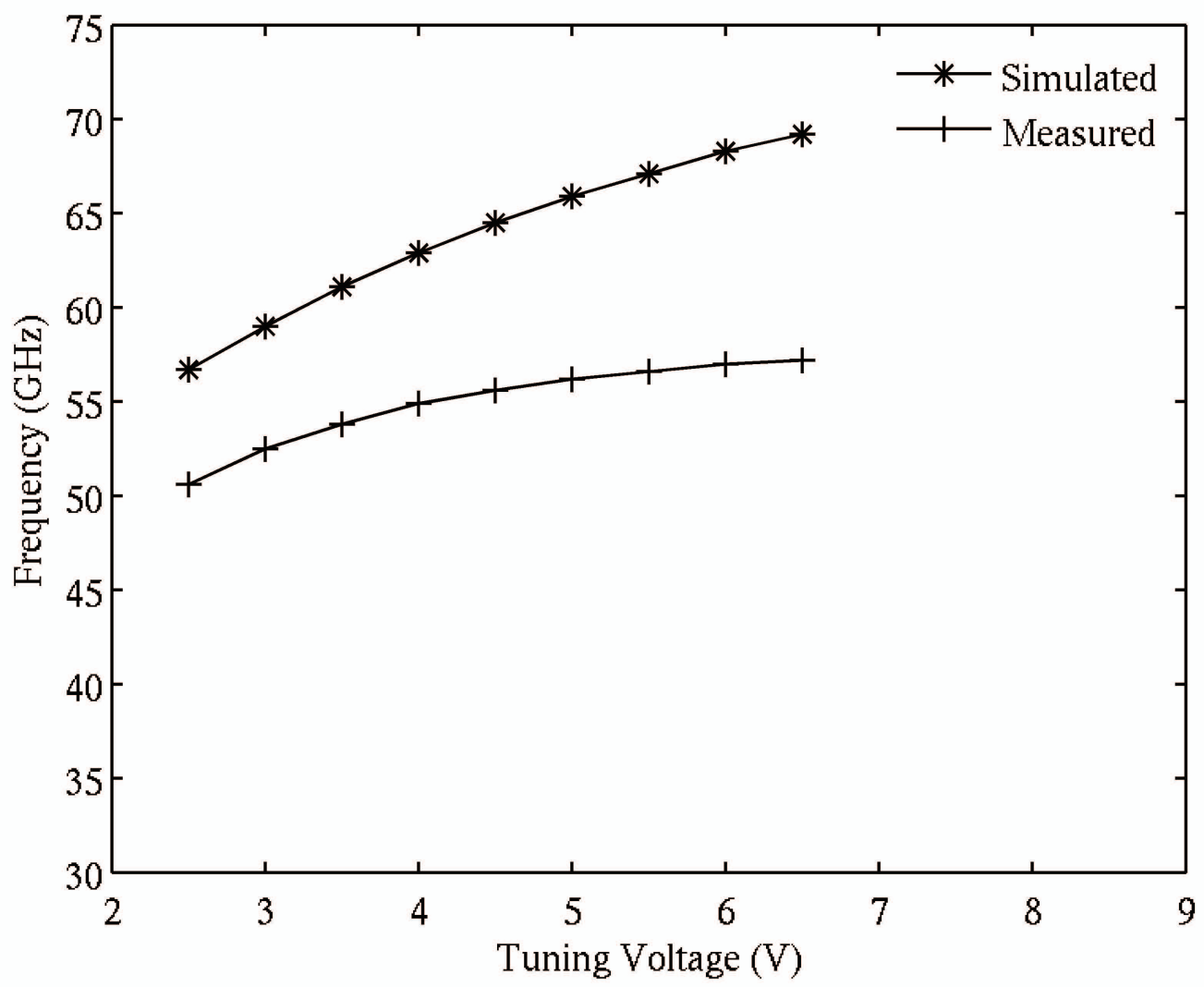

